Reprod. Nutr. Dévelop., 1982, 22 (1 A), 65-73.

\title{
Relation entre l'espace de diffusion de l'eau lourde mesurée in vivo et le volume hydrique corporel des bovins en croissance
}

\section{J. ROBELIN}

avec la collaboration technique de Christiane BARBOIRON et Roland JAILLER

Laboratoire de la Production de Viande, I.N.R.A.

Theix, St-Genès-Champanelle 63110 Beaumont, France.

Summary. Relation in vivo between the dilution space of deuteriated water and body water in growing cattle.

Twenty-one Charolais and 21 Friesian bulls were infused with deuteriated water $(0.6 \mathrm{~g} / \mathrm{kg}$ body weight). Blood samples were collected 6,9 and 24 hours after infusion. The dilution space was calculated by either the zero-time extrapolation method $\left(\mathrm{EDC}_{0}\right)$ or by 6hour marker concentration in blood water $\left(E D C_{6}\right)$. The animals were slaughtered and their empty body water (ECV) and total body water (ECTC) measured.

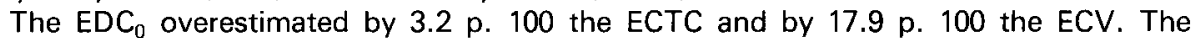
standard error of ECTC estimation was lower (2.0 p. 100 of ECTC) than that of ECV (4.3 p. 100 of ECV). The EDC ${ }_{6}$ overestimated by 4.7 p. 100 the ECTC and was less related to it (SE of estimation $=2.4$ p. $100 \mathrm{ECTC}$ ). It was concluded that the zero-time extrapolation method of determining the dilution space was preferable.

The dilution space of deuteriated water was a fairly good estimate of total body water. Taking into account the close relationship between body water and body lipids, the dilution space measurement could be used to assess the chemical composition of live animals.

\section{Introduction.}

La mesure directe de la composition corporelle des animaux par la méthode des abattages suivis du broyage du corps entier et de son analyse chimique est très coûteuse surtout chez les bovins. C'est une mesure de référence qui ne peut être réalisée que sur un nombre limité d'animaux. L'estimation de la composition corporelle, grâce à des critères simples mesurés sur l'animal après abattage (Robelin et Geay, 1978), permet de réduire considérablement le coût de la mesure. Cette méthode permet d'estimer avec précision la composition des animaux et la composition moyenne du croît de lots d'animaux comparables. Mais elle exige des abattages à différents stades successifs et ne permet pas d'estimer la composition du croît au niveau individuel. C'est pourquoi, nous avons tenté de mettre au point une méthode d'estimation de la composition corporelle in vivo à partir de l'espace de diffusion de l'eau lourde (cf. revue bibliographique, Robelin, 
1973). Cette méthode donne de bons résultats chez les ovins (Foot et Greenhagh, 1970 ; Robelin, 1977 ; Cowan et al., 1979 ; Tissier, résultats en cours de publication). Elle a déjà été utilisée pour estimer la composition corporelle des bovins (Webster et al., 1978 ; Chigaru et Topps, 1981), mais sa mise au point, c'est-àdire la comparaison sur les mêmes animaux entre l'espace de diffusion de l'eau lourde (ou de l'eau tritiée) et le volume hydrique réel mesuré après abattage, n'a été réalisée jusqu'alors que sur un nombre limité d'animaux (Canergie et Tulloh, 1968 : 26 animaux; Little et Morris, $1972: 9$ animaux; Crabtree, Houseman et Kay, $1974: 12$ animaux). C'est pourquoi nous avons effectué cette comparaison sur un nombre important de bovins en croissance. Cet article a pour objet de décrire avec précision les méthodes utilisées et d'analyser la relation entre l'espace de diffusion de l'eau lourde et le volume hydrique corporel des animaux.

\section{Matériel animal et analyse critique des méthodes utilisées.}

Animaux. - Lors d'une étude préliminaire, on a analysé la diffusion de l'eau lourde dans l'eau corporelle de 9 jeunes bovins mâles Limousins, âgés de 13 à 19 mois et dont le poids vif variait de 400 à $650 \mathrm{~kg}$.

Ensuite, on a mesuré simultanément l'espace de diffusion de l'eau lourde et le volume hydrique réel du corps entier de 42 jeunes bovins mâles de races Frisonne $(n=21)$ et Charolaise $(n=21)$ abattus à différents stades de maturité $(15,25,35,45$ et 55 p. 100 du poids vif adulte estimé) au cours d'expériences à caractère nutritionnel.

Les animaux de race Charolaise ont été élevés sous la mère jusqu'à l'âge de 9 mois, à un poids vif compris entre 250 et $300 \mathrm{~kg}$. Les animaux Frisons ont été sevrés précocement à l'âge de 3 mois et ont reçu à volonté jusqu'à l'âge de 9 mois une ration comportant 40 p. 100 d'aliments concentrés et 60 p. 100 de fourrage. Passé l'âge de 9 mois, les animaux des deux génotypes ont reçu à volonté la même ration comportant 80 p. 100 d'aliments concentrés et 20 p. 100 de fourrage. Leur croissance pondérale moyenne a été supérieure à $1 \mathrm{~kg}$ par jour.

Le jour de l'injection d'eau lourde, les animaux ont reçu normalement leurs deux repas journaliers, respectivement à 7 et $16 \mathrm{~h}$ environ, mais $\mathrm{n}^{\prime}$ ont pas été alimentés le lendemain matin, juste avant l'abattage ; ils ont eu accès à un abreuvoir automatique jusqu'à l'abattage.

Injection du marqueur, prélèvements de sang et dosage de l'eau lourde. L'injection d'eau lourde (CEA-CEN-SACLAY ; pureté : 99,8\%) a été effectuée le matin, entre $8 \mathrm{~h}$ et $10 \mathrm{~h}$, soit $2 \mathrm{~h}$ en moyenne après la distribution du premier repas. Pour chaque animal, le marqueur, contenu dans des seringues de $50 \mathrm{ml}$, a été injecté à raison de $0,6 \mathrm{~g}$ par $\mathrm{kg}$ de poids vif de l'animal, par voie jugulaire à l'aide d'un cathéter.

Après l'injection du marqueur, le cathéter a été rincé avec une solution isotonique. Le poids d'eau lourde injecté a été calculé par différence entre le poids des seringues pleines (pesées ensemble) et le poids de ces seringues (non rincées) après l'injection. 
On a ensuite prélevé 3 échantillons de sang, respectivement 6,9 et $24 \mathrm{~h}$ après l'injection, les premiers essais réalisés sur les animaux Limousins ayant montré que la durée nécessaire à la diffusion du marqueur était voisine de $6 \mathrm{~h}$. Une durée comparable a d'ailleurs été observée précédemment chez les bovins avec I'eau lourde ou l'eau tritiée (Little et Morris, 1972 ; Crabtree, Houseman et Kay, 1974).

L'extraction de l'eau du sang prélevé après l'injection et le dosage de l'eau lourde ont été effectués selon la méthode décrite précédemment (Robelin, 1977 ; Tissier et al., 1978). On a effectué 4 dosages par échantillon. La précision sur cette mesure est alors égale à 0,5 p. 100 en valeur relative.

Analyse de la diffusion du marqueur et calcul de son espace de diffusion. L'évolution après l'injection de la concentration du marqueur dans l'eau du sang peut être séparée en deux phases comme nous l'avons précisé précédemment (Robelin, 1973, 1977). Immédiatement après l'injection, la concentration du marqueur exprimée en logarithme décroît en fonction du temps, rapidement et curvilinéairement. Cette phase correspond à la diffusion du marqueur dans la totalité de l'eau corporelle. Cette diffusion étant terminée, la concentration du marqueur est identique dans l'ensemble de l'eau corporelle; le marqueur est alors éliminé au rythme du renouvellement de cette dernière. La concentration du marqueur dans l'eau du sang, toujours exprimée en logarithme, décroît alors linéairement, ce qui traduit une relation du type : $\mathrm{C}=\mathrm{Co} \mathrm{e}^{-\mathrm{bt}}$.

On a calculé, pour chaque animal, les coefficients de cette relation (Co et b) à l'aide des concentrations en eau lourde observées 6,9 et $24 \mathrm{~h}$ après l'injection ; on a calculé enfin l'espace de diffusion du marqueur $\left(E D C_{0}\right)$, rapport entre la quantité d'eau lourde injectée et la concentration initiale théorique (Co).

A titre de comparaison, on a également calculé l'espace de diffusion $\left(E C_{6}\right)$ à partir de la concentration du marqueur mesurée $6 \mathrm{~h}$ après l'injection, c'est-à-dire après que la diffusion soit terminée. C'est cette méthode qui est le plus souvent utilisée dans les travaux rapportés dans la bibliographie.

Détermination du poids vif des animaux. - Les animaux ont été pesés régulièrement au cours de leur croissance à $14 \mathrm{~h}$, c'est-à-dire entre la distribution des deux repas quotidiens $(7 \mathrm{~h}$ et $16 \mathrm{~h}$ ). En particulier, ils ont été pesés 6 fois au cours du dernier mois, dont 2 fois les 2 jours précédant l'injection. On a estimé par régression linéaire à partir de ces 6 pesées le poids « moyen » des animaux (PM) au jour de l'injection. En outre, les animaux ont été pesés au moment de l'injection (PI), c'est-à-dire 1 à $2 \mathrm{~h}$ après la distribution du repas du matin, et le lendemain, juste avant l'abattage (PA), c'est-à-dire $18 \mathrm{~h}$ environ après la distribution du dernier repas. Ces trois valeurs du poids vif sont significativement différentes $(P<0,05)$. Le poids "moyen " (PM), c'est-à-dire le poids de l'animal estimé par régression, est supérieur de 1,1 p. 100 au poids mesuré au moment de l'injection (PI) et de 2,6 p. 100 supérieur au poids mesuré à l'abattage (PA).

Abattage et mesure de la composition corporelle. - Les animaux ont été abattus le matin, $24 \mathrm{~h}$ après l'injection d'eau lourde. On a mesuré le poids vif à 
I'abattage, le poids et la teneur en eau des contenus digestifs, ainsi que la teneur en eau du corps vide (corps entier - contenus digestifs) selon la méthode décrite précédemment (Robelin, Geay et Béranger, 1979).

On a déterminé ainsi la quantité d'eau contenue dans le corps vide (ECV), celle contenue dans les digestas (ECD) et l'eau corporelle totale (ECTA = ECV + ECD) (fig. 1). Ce volume hydrique mesuré à l'abattage correspond à l'animal privé de nourriture depuis $18 \mathrm{~h}$ environ. II peut donc difficilement être comparé à l'espace de diffusion de l'eau lourde, qui est estimé à partir des caractéristiques de diffusion du marqueur pendant $24 \mathrm{~h}$ et qui doit par conséquent être

TABLEAU 1

Poids vif et compartiments hydriques corporels des animaux (moyenne et écart type de la moyenne)

\begin{tabular}{|c|c|c|c|c|c|c|c|c|c|c|c|}
\hline \multicolumn{3}{|c|}{ Animaux (1) } & \multicolumn{4}{|c|}{ Poids vif $(\mathrm{kg})(2)$} & \multicolumn{3}{|c|}{ Eau corporelle (kg) (3) } & \multicolumn{2}{|c|}{$\begin{array}{c}\text { Espace de } \\
\text { diffusion }(\mathrm{kg})(4)\end{array}$} \\
\hline Race & Stade & Nombre & PM & $\mathrm{PI}$ & PA & PVV & ECV & ECTA & ЕСТС & $\mathrm{EDC}_{0}$ & $\mathrm{EDC}_{6}$ \\
\hline 1 & 15 & 5 & $\begin{array}{l}128 \\
(3)\end{array}$ & $\begin{array}{l}129 \\
(4)\end{array}$ & $\begin{array}{l}124 \\
(3)\end{array}$ & $\begin{array}{l}107 \\
(2)\end{array}$ & $\begin{array}{r}75 \\
(1)\end{array}$ & $\begin{array}{r}90 \\
(2)\end{array}$ & $\begin{array}{r}94 \\
(2)\end{array}$ & $\begin{array}{l}100 \\
(2)\end{array}$ & $\begin{array}{l}102 \\
(3)\end{array}$ \\
\hline 1 & 25 & 4 & $\begin{array}{l}228 \\
(2)\end{array}$ & $\begin{array}{l}227 \\
(3)\end{array}$ & $\begin{array}{l}214 \\
(3)\end{array}$ & $\begin{array}{l}185 \\
(2)\end{array}$ & $\begin{array}{l}126 \\
(2)\end{array}$ & $\begin{array}{l}151 \\
(3)\end{array}$ & $\begin{array}{l}163 \\
(1)\end{array}$ & $\begin{array}{l}171 \\
(2)\end{array}$ & $\begin{array}{l}172 \\
(2)\end{array}$ \\
\hline 1 & 35 & 4 & $\begin{array}{l}321 \\
(3)\end{array}$ & $\begin{array}{l}315 \\
(2)\end{array}$ & $\begin{array}{l}314 \\
(3)\end{array}$ & $\begin{array}{l}279 \\
(2)\end{array}$ & $\begin{array}{l}181 \\
(3)\end{array}$ & $\begin{array}{l}211 \\
(3)\end{array}$ & $\begin{array}{l}217 \\
(3)\end{array}$ & $\begin{array}{l}227 \\
(2)\end{array}$ & $\begin{array}{l}231 \\
(3)\end{array}$ \\
\hline 1 & 45 & 4 & $\begin{array}{l}396 \\
(10)\end{array}$ & $\begin{array}{l}387 \\
(10)\end{array}$ & $\begin{array}{l}386 \\
(10)\end{array}$ & $\begin{array}{l}350 \\
(10)\end{array}$ & $\begin{array}{l}217 \\
(4)\end{array}$ & $\begin{array}{l}248 \\
(5)\end{array}$ & $\begin{array}{l}256 \\
(5)\end{array}$ & $\begin{array}{l}263 \\
(7)\end{array}$ & $\begin{array}{l}268 \\
(7)\end{array}$ \\
\hline 1 & 55 & 4 & $\begin{array}{l}506 \\
(23)\end{array}$ & $\begin{array}{l}500 \\
(20)\end{array}$ & $\begin{array}{l}500 \\
(22)\end{array}$ & $\begin{array}{l}451 \\
(21)\end{array}$ & $\begin{array}{l}257 \\
(9)\end{array}$ & $\begin{array}{l}299 \\
(10)\end{array}$ & $\begin{array}{l}304 \\
(11)\end{array}$ & $\begin{array}{l}317 \\
(12)\end{array}$ & $\begin{array}{l}323 \\
(13)\end{array}$ \\
\hline 2 & 15 & 5 & $\begin{array}{r}160 \\
(8)\end{array}$ & $\begin{array}{r}159 \\
(8)\end{array}$ & $\begin{array}{l}160 \\
(8)\end{array}$ & $\begin{array}{l}142 \\
(7)\end{array}$ & $\begin{array}{l}101 \\
(5)\end{array}$ & $\begin{array}{l}118 \\
(6)\end{array}$ & $\begin{array}{l}117 \\
(6)\end{array}$ & $\begin{array}{l}123 \\
(6)\end{array}$ & $\begin{array}{l}127 \\
(6)\end{array}$ \\
\hline 2 & 25 & 4 & $\begin{array}{l}296 \\
(4)\end{array}$ & $\begin{array}{l}291 \\
(6)\end{array}$ & $\begin{array}{l}284 \\
(6)\end{array}$ & $\begin{array}{l}253 \\
(6)\end{array}$ & $\begin{array}{r}172 \\
(2)\end{array}$ & $\begin{array}{l}199 \\
(2)\end{array}$ & $\begin{array}{l}209 \\
(1)\end{array}$ & $\begin{array}{l}213 \\
(3)\end{array}$ & $\begin{array}{l}217 \\
(2)\end{array}$ \\
\hline 2 & 35 & 4 & $\begin{array}{l}386 \\
(6)\end{array}$ & $\begin{array}{l}381 \\
(7)\end{array}$ & $\begin{array}{l}376 \\
(6)\end{array}$ & $\begin{array}{l}344 \\
(5)\end{array}$ & $\begin{array}{l}225 \\
(4)\end{array}$ & $\begin{array}{l}253 \\
(5)\end{array}$ & $\begin{array}{l}261 \\
(5)\end{array}$ & $\begin{array}{r}266 \\
(5)\end{array}$ & $\begin{array}{l}271 \\
(5)\end{array}$ \\
\hline 2 & 45 & 4 & $\begin{array}{l}497 \\
(7)\end{array}$ & $\begin{array}{l}491 \\
(5)\end{array}$ & $\begin{array}{l}489 \\
(5)\end{array}$ & $\begin{array}{l}448 \\
(5)\end{array}$ & $\begin{array}{l}289 \\
(7)\end{array}$ & $\begin{array}{l}324 \\
(8)\end{array}$ & $\begin{array}{l}331 \\
(10)\end{array}$ & $\begin{array}{l}340 \\
(11)\end{array}$ & $\begin{array}{l}343 \\
(10)\end{array}$ \\
\hline 2 & 55 & 4 & $\begin{array}{r}580 \\
(9)\end{array}$ & $\begin{array}{l}578 \\
(5)\end{array}$ & $\begin{array}{l}565 \\
(4)\end{array}$ & $\begin{array}{l}516 \\
(2)\end{array}$ & $\begin{array}{l}329 \\
(6)\end{array}$ & $\begin{array}{l}372 \\
(8)\end{array}$ & $\begin{array}{l}385 \\
\text { (9) }\end{array}$ & $\begin{array}{l}398 \\
(10)\end{array}$ & $\begin{array}{l}402 \\
(8)\end{array}$ \\
\hline
\end{tabular}

(1) Race 1 = Frisonne, 2 = Charolaise. Stade d'abattage exprimé en pourcentage du poids adulte des animaux.

(2) $\mathrm{PM}=$ poids vif " moyen » calculé par régression linéaire à partir de 6 pesées antérieures à l'abattage effectuées vers $14 \mathrm{~h}$, c'est-à-dire entre la distribution des deux repas quotidiens, PI = void vif " injection » mesuré au moment de l'injection, environ $2 \mathrm{~h}$ après la distribution du premier repas quotidien. PA = poid vif « abattage " mesuré juste avant l'abattage, c'est-à-dire $18 \mathrm{~h}$ après la distribution du dernier repas. PVV = poids vif " vide » mesuré après l'abattage. Poids du corps entier diminué de celui du contenu digestif.

(3) ECV = eau du corps vide, mesurée par dessiccation après l'abattage. ECTA = eau corporelle totale (corps vide et contenu digestif) mesurée par dessiccation après l'abattage, ECTC = eau corporelle totale corrigée, calculée à partir de ECTA et de la différence de poids PM-PA (cf. Matériel et méthodes).

(4) $\mathrm{EDC}_{0}=$ Espace de diffusion de l'eau lourde calculé à partir de la concentration initiale du marqueur déterminée par régression. $\mathrm{EDC}_{6}=$ Espace de diffusion de l'eau lourde calculé à partir de la concentration du marqueur mesurée $6 \mathrm{~h}$ après l'injection. 
représentatif de l'état moyen de l'animal au cours d'un cycle d'alimentation de $24 \mathrm{~h}$. On a donc calculé un volume d'eau du contenu digestif corrigé (ECDC) en tenant compte de la différence $(\Delta P)$ de poids vif des animaux entre le poids vif " moyen " $(\mathrm{PM})$ déterminé par régression, et le poids vif mesuré avant l'abattage (PA) (fig. 1). A cet effet, on a affecté à cette différence de poids ( $\triangle P=P M-P A)$ correspondant à du contenu digestif, la teneur en eau $(X)$ du contenu digestif mesurée après l'abattage ( $E C D C=E C D+\triangle P \times X)$. On a calculé ensuite le volume hydrique corporel total corrigé (ECTC $=E C V+E C D C$ ).

Les valeurs moyennes par génotype et par stade d'abattage de ces différentes mesures sont rapportées au tableau 1.

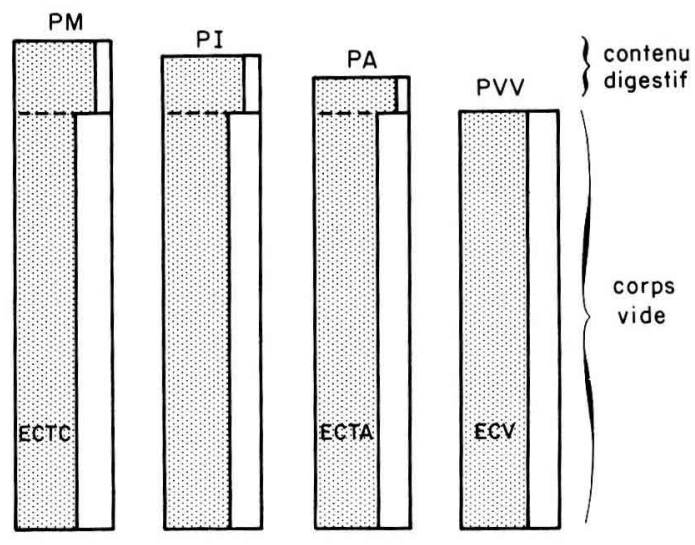

FIG. 1. - Représentation schématique des différentes valeurs du poids vif mesurées sur les animaux, et des compartiments hydriques correspondants (zones pointillées) (voir légende tabl. 1). PM : poids moyen ; PI : poids injection : PA : poids abattage ; PVV : poids vif vide. ECTC : eau corporelle totale corrigée; ECTA : eau corporelle totale abattage; ECV : eau du corps vide.

\section{Résultats - Discussion.}

\section{Diffusion du marqueur dans l'eau corporelle.}

La concentration (C) de l'eau lourde corporelle varie en fonction du temps $(t)$ selon une relation du type : $C=C o e^{-b t}$. La valeur moyenne de la pente $b$, sur l'ensemble des 42 animaux Charolais et Frisons est égale à 0,00332 $( \pm 0,00027)$; la vitesse de renouvellement de l'eau corporelle chez ces bovins en croissance est donc voisine de 0,3 p. 100 (p. 100 du volume hydrique corporel total) par heure, soit 7,6 p. 100 par jour, ce qui représente environ $18 \mathrm{~kg} \mathrm{~d}$ 'eau pour un bovin pesant $400 \mathrm{~kg}$. Ce renouvellement qui correspond à une demi-vie biologique du deutérium de 8 jours est comparable à celui qui a été observé précédemment chez les bovins avec le tritium (4 à 9 jours selon les auteurs; cf. revue de Robelin, 1973). Cette vitesse de renouvellement n'est pas significativement différente selon la race des animaux $(b=0,00311 \pm 0,0035$ et $0,00352 \pm 0,00042$ respectivement chez les Frisons et les Charolais), ou selon leur stade de développement $(15,25,35,45$ ou 55 p. 100 du poids adulte). 
Relation entre l'espace de diffusion de l'eau lourde et le volume hydrique corporel réel.

On a mesuré à l'abattage trois compartiments hydriques corporels représentés sur la figure 1 et qui sont respectivement l'eau du corps vide (ECV) (sans le contenu digestif), l'eau corporelle totale au moment de l'abattage (ECTA) et enfin l'eau corporelle totale corrigée (ECTC) ; on a mesuré également l'espace de diffusion de l'eau lourde, déterminé à partir de la concentration initiale du marqueur (espace de diffusion $E D C_{0}$ ) ou à partir de la concentration observée $6 \mathrm{~h}$ après I'injection (espace de diffusion $\left.E^{2} C_{6}\right)$. Les relations $\left(Y=b_{0}+b_{1} X\right)$ entre l'un des trois compartiments hydriques corporels $(Y ; \mathrm{kg})$ et l'espace de diffusion $\mathrm{EDC}_{O}(\mathrm{X} ; \mathrm{kg})$ ont un terme constant $\left(\mathrm{b}_{0}\right)$ non significativement différent de zéro $(P<0,01)$; en d'autres termes, le fait d'imposer la valeur zéro à $b_{0}$ n'entraîne pas d'accroissement significatif de l'écart type résiduel de la régression $Y=b_{0}+b_{1} X$ (tabl. 2). Par ailleurs, les coefficients $b_{1}$ ne sont pas significativement différents selon le génotype des animaux, Charolais ou Frison. Enfin, I'espace de diffusion $\mathrm{EDC}_{0}$ est supérieur aux trois compartiments hydriques corporels. C'est l'eau du corps vide (ECV) qui est le plus éloigné de $E C_{0}$ $\left(b_{1}=0,821 \pm 0,005\right)$ et qui lui est le moins étroitement relié lécart type résiduel $=$ $8,31 \mathrm{~kg}$ soit 4,3 p. 100 en valeur relative) ; au contraire, c'est le volume hydrique total corrigé $(E C T C)$ qui en est le plus proche $\left(b_{1}=0,968 \pm 0,003\right)$ et qui lui est le plus étroitement relié lécart type résiduel $=4,63 \mathrm{~kg}$ soit $2 \mathrm{p}$. 100 en valeur relative). L'espace de diffusion de l'eau lourde EDC $E_{0}$ est donc supérieur de 3,2 p. 100 au volume hydrique total corrigé.

\section{TABLEAU 2}

Relations entre l'espace de diffusion de l'eau lourde $(\mathrm{X} ; \mathrm{kg})$ et le volume hydrique corporel $(\mathrm{Y} ; \mathrm{kg})$ des jeunes bovins frisons et charolais: $Y=b_{0}^{\prime}+b_{1}^{\prime} X \pm s y x ; Y=b_{1} X \pm s y x_{0}$

\begin{tabular}{|c|c|c|c|c|c|c|}
\hline Animaux & $Y(1)$ & $x(1)$ & $b_{1}$ & $\mathbf{s b}_{1}$ & syx $_{0}$ & syx \\
\hline $\begin{array}{l}\text { Frisons } \\
\{n=21\}\end{array}$ & $\begin{array}{l}\text { ECTC } \\
\text { ECTA } \\
\text { ECV } \\
\text { ECTC }\end{array}$ & $\begin{array}{l}\mathrm{EDC}_{0} \\
\mathrm{EDC}_{0} \\
\mathrm{EDC}_{0} \\
\mathrm{EDC}_{6}\end{array}$ & $\begin{array}{l}0,961 \\
0,932 \\
0,801 \\
0,944\end{array}$ & $\begin{array}{l}0,004 \\
0,006 \\
0,007 \\
0,005\end{array}$ & $\begin{array}{l}4,64 \\
6,18 \\
7,59 \\
5,32\end{array}$ & $\begin{array}{l}4,51 \\
5,28 \\
6,01 \\
5,27\end{array}$ \\
\hline $\begin{array}{l}\text { Charolais } \\
(\mathrm{n}=21)\end{array}$ & $\begin{array}{l}\text { ECTC } \\
\text { ECTA } \\
\text { ECV } \\
\text { ECTC }\end{array}$ & $\begin{array}{l}\mathrm{EDC}_{0} \\
\mathrm{EDC}_{0} \\
\mathrm{EDC}_{0} \\
\mathrm{EDC}_{6}\end{array}$ & $\begin{array}{l}0,972 \\
0,943 \\
0,833 \\
0,960\end{array}$ & $\begin{array}{l}0,003 \\
0,004 \\
0,006 \\
0,004\end{array}$ & $\begin{array}{l}4,41 \\
4,68 \\
7,22 \\
5,05\end{array}$ & $\begin{array}{l}4,40 \\
4,52 \\
7,19 \\
4,82\end{array}$ \\
\hline $\begin{array}{l}\text { Ensemble des animaux } \\
\qquad(\mathrm{n}=42)\end{array}$ & $\begin{array}{l}\text { ECTC } \\
\text { ECTA } \\
\text { ECV } \\
\text { ECTC }\end{array}$ & $\begin{array}{l}\mathrm{EDC}_{0} \\
\mathrm{EDC}_{0} \\
\mathrm{EDC}_{0} \\
\mathrm{EDC}_{6}\end{array}$ & $\begin{array}{l}0,968 \\
0,939 \\
0,821 \\
0,954\end{array}$ & $\begin{array}{l}0,003 \\
0,003 \\
0,005 \\
0,003\end{array}$ & $\begin{array}{l}4,63 \\
\mathbf{5 , 5 1} \\
\mathbf{8 , 3 1} \\
\mathbf{5 , 4 8}\end{array}$ & $\begin{array}{l}4,55 \\
5,35 \\
7,47 \\
5,24\end{array}$ \\
\hline
\end{tabular}

(1) ECV = eau du corps vide, mesurée par dessiccation après abattage. ECTA = eau corporelle totale (corps vide et contenu digestif) mesurée par dessiccation après l'abattage. ECTC = eau corporelle totale corrigée, calculée à partir de ECTA et de la différence de poids PM - PA (cf. Matériel et méthodes). $\mathrm{EDC}_{0}=$ Espace de l'eau lourde calculé à partir de la concentration initiale du marqueur déterminée par régression. $\mathrm{EDC}_{6}=$ Espace de diffusion de l'eau lourde calculé à partir de la concentration du marqueur mesurée $6 \mathrm{~h}$ après l'ínjection. 
L'espace de diffusion $\mathrm{EDC}_{6}$ déterminé à partir de la concentration du marqueur $6 \mathrm{~h}$ après l'injection est supérieur à $E D C_{0}$; il dépasse de 4,7 p. 100 (tabl. 2) le volume hydrique total corrigé, et lui est relié moins étroitement lécart type résiduel $=5,48 \mathrm{~kg}$ ) que l'espace de diffusion $\mathrm{EDC}_{0}$.

Cette surestimation du volume hydrique corporel par l'espace de diffusion est un résultat quasi général (cf. revue de Robelin, 1973), quels que soient l'espèce animale et le marqueur utilisés (eau lourde ou eau tritiée). Elle atteint 10 à 15 p. 100 du volume hydrique chez les bovins d'après les résultats de Canergie et Tulloh (1968) et ceux de Crabtree, Houseman et Kay (1974). On peut l'expliquer en partie par un échange d'hydrogène marqué avec les protéines et les lipides corporels et par une rétention de cet hydrogène dans l'eau liée du muscle ; cependant, ces échanges ne peuvent expliquer qu'une différence de 1 à 4 p. 100 entre l'espace de diffusion et le volume hydrique (Robelin, 1973).

Cette différence est également liée à la méthode de calcul de l'espace de diffusion; ainsi, comme nous venons de le voir, elle est plus élevée lorsque l'on calcule ce dernier à partir de la concentration plasmatique du marqueur $6 \mathrm{~h}$ après l'injection, qui est plus faible que la concentration initiale déterminée par régression ; Foot, Skedd et Mc Farlane (1979) obtiennent également une différence d'environ 6 p. 100 en valeur relative, chez la brebis, entre l'espace de diffusion de l'eau tritiée calculé par extrapolation et l'espace de diffusion calculé à partir de la concentration du marqueur mesurée $6 \mathrm{~h}$ après l'injection.

Enfin, la différence entre l'espace de diffusion et le volume hydrique corporel dépend des conditions dans lesquelles ce dernier est mesuré ; à cet égard, il semble que le volume hydrique corrigé tel que nous l'avons déterminé soit plus représentatif du volume hydrique "moyen » des animaux, que l'eau corporelle totale mesurée au moment de l'abattage, c'est-à-dire $18 \mathrm{~h}$ après la distribution du dernier repas ; en effet, c'est ce volume corrigé qui est le plus étroitement relié à l'espace de diffusion. Cette correction que nous avons effectuée repose sur I'hypothèse que l'eau corporelle totale subit des fluctuations au cours de la journée chez les bovins; cette hypothèse n'est évidemment pas vérifiable directement ; elle repose néanmoins sur des faits concordants ; on a observé chez des bovins pesant $485 \mathrm{~kg}$ au cours des deux journées consécutives, un accroissement de 10 à $15 \mathrm{~kg}$ du poids vif entre le matin avant la distribution du premier repas et le soir, $2 \mathrm{~h}$ après la distribution du second repas (résultats non publiés obtenus sur 12 animaux) ; cet accroissement est largement supérieur à la quantité de matière sèche ingérée $(7$ à $8 \mathrm{~kg})$, diminuée de la matière sèche excrétée $(1 \mathrm{à} 2 \mathrm{~kg}$ ) par les animaux durant cette période. II correspond donc bien à un accroissement temporaire de l'eau corporelle totale, et très probablement de l'eau du contenu digestif.

La précision de la relation entre l'espace de diffusion $\mathrm{EDC}_{0}$ et le volume hydrique total corrigé est très bonne ; l'écart-type résiduel de la régression $(4,63 \mathrm{~kg})$ représente 2,0 p. 100 du volume hydrique corporel. Cette précision est comparable à celle observée chez différentes espèces, de 2 à 4 p. 100 selon les auteurs (Robelin, 1973). Chez les bovins, des valeurs de 2,8 à 4,5 p. 100 ont été obtenues par Little et Morris (1972) et Crabtree, Houseman et Kay (1974). Une valeur de 2 p. 100 doit correspondre au minimum que l'on puisse atteindre compte tenu des erreurs inhérentes à la détermination de l'espace de diffusion et du volume hydri- 
que corporel. Certaines d'entre elles sont quantifiables ; ainsi, la précision de la mesure de la concentration en eau lourde de l'eau plasmatique et celle de la mesure de la teneur en eau du corps entier à l'abattage, sont voisines de 0,5 p. 100 en valeur relative ; en revanche, d'autres erreurs, qui résultent des fluctuations de l'eau corporelle au cours de la journée ne peuvent pas être quantifiées. On peut penser qu'elles sont d'autant plus faibles que le rythme de vie et d'alimentation des animaux est moins perturbé, et que les conditions de mesure de l'espace de diffusion (heures de l'injection et des prélèvements de sang) sont mieux standardisées. A cet égard, le fait de calculer l'espace de diffusion du marqueur par régression à partir de plusieurs prélèvements de sang permet certainement de tamponner ces variations.

En conclusion, l'eau lourde est un bon marqueur de l'eau corporelle totale chez les bovins ; son espace de diffusion permet d'estimer in vivo avec précision (environ 2 p. 100 en valeur relative) le volume hydrique corporel des animaux. Compte tenu de la relation statistique bien connue entre l'eau et les lipides corporels (Moulton, 1923 ; Robelin et Daenicke, 1980 ; Robelin et Thériez, 1981), cette mesure permet d'estimer in vivo l'ensemble de la composition chimique corporelle des animaux. Cette application de la mesure de l'espace de diffusion de l'eau lourde sera abordée dans une publication ultérieure.

Le coût élevé de la mesure de l'espace de diffusion de l'eau lourde (environ $1 \mathrm{~F}$ par kg de poids vif des animaux en 1981) fait que cette mesure est surtout réservée aux animaux de petite taille tels que les ovins. Cette mesure conserve cependant son intérêt chez les bovins, pour effectuer une cinétique sur les mêmes animaux, ou lorsque la méthode des abattages serait trop onéreuse (vaches adultes en production), voire inenvisageable (animaux en contrôle de performance). Un essai d'application de la mesure est en cours de réalisation sur des taureaux destinés à la reproduction et nous poursuivons actuellement la mise au point de la méthode sur des vaches adultes.

Reçu en août 1980.

Accepté en août 1981.

\section{Références}

CANERGIE A. B., TULLOH N. M., 1968. The in vivo determination of body water space in cattle using the tritium dilution technique. Proc. aust. Soc. anim. Prod., 7, 308-313.

CHIGARU P. R. N., TOPPS J. H., 1981. The composition of body weight changes in underfed lactating beef cows. Anim. Prod., 32, 95-103.

COWAN R. T., ROBINSON J. J., GREENHALGH J. F. D., Mc HATIE I., 1979. Body composition changes in lacting ewes estimated by serial slaughter and deuterium dilution. Anim. Prod., 29, 81-90.

CRABTREe R. M., HOUSEMAN R. A., KAY M., 1974. The estimation of body composition in beef cattle by deuterium oxide dilution. Proc. Nut. Soc., 33, 74 A-75 A.

FOOT J. Z., GREENHALGH J. F. D., 1970. The use of deuterium oxyde space to determine the amount of body fat in pregnant Blackface ewes. Br. J. Nutr., 24, 815-825.

FOOT J. Z., SKEDD E., MC FARLANE D. N., 1979. Body composition in lactating sheep and its indirect measurement in the live animal using tritiated water. J. agric. Sci., 92, 68-81. 
LITTLE D. A., MORRIS J. G., 1972. Prediction of the body composition of live cattie. J. agric. Sci, 78, 505-508.

MOULTON, 1923. Age and chemical development in mammals. J. biol. Chem., 57, 79-97.

ROBELIN J., 1973. Estimation de la composition corporelle des animaux à partir de l'espace de diffusion de l'eau marquée. Ann. Biol. anim. Bioch. Biophys., 13, 285-305.

ROBELIN J., 1977. Estimation in vivo de la composition corporelle des agneaux à partir de l'espace de diffusion de l'eau lourde. Ann. Biol. anim. Bioch. Biophys., 17, 95-105.

ROBELIN J., DAENICKE R., 1980. Variations of net requirements for cattle growth with liveweight, liveweight gain, breed and sex. Ann. Zootech., 29, $\mathrm{n}^{\circ}$ h.s., 99-118.

ROBELIN J., GEAY Y., 1978. Estimation de la composition chimique du corps entier des bovins à partir du poids des dépôts adipeux totaux. Ann. Zootech., 27, 159-167.

ROBELIN J., GEAY Y., BÉRANGER C., 1979. Evolution de la composition corporelle des jeunes bovins mâles entiers de race Limousine entre 9 et 19 mois. II. Composition chimique et valeur calorifique. Ann. Zootech., 28, 191-208.

ROBELIN J., THÉRIEZ M., 1981. Fixation de protéines chez les ruminants: évolution en fonction du poids des animaux et variations selon la race, le sexe ou le niveau des apports alimentaires. Reprod. Nutr. Dévelop., 21, 335-353.

TISSIER M., ROBELIN J., PURROY A., GEAY Y., 1978. Extraction et dosage automatique rapide de l'eau lourde dans les liquides biologiques. Ann. Biol. anim. Bioch. Biophys., 18, 1223-1228.

Webster A. J. F., SMITH J. S., CRABTREE R. M., MOLlison G. S., 1978. Prediction of the energy requirement for growth in beef cattle. 2 . Hereford $\times$ British Friesian steers given dried grass or barley. Anim. Prod., 23, 329-340. 